\title{
EdiMM: uma ferramenta educacional para construção de textos multissemióticos com interação multimodal e para multidispositivos
}

\author{
André C. da Silva ${ }^{1,2}$, Flávia L. Arantes², Fernanda M. P. Freire², Daniel R. S. \\ Silva ${ }^{1}$ \\ ${ }^{1}$ Grupo Mobilidade e Novas Tecnologias de Interação - Instituto Federal de São Paulo \\ câmpus Hortolândia (IFSP) \\ Hortolândia -SP - Brasil \\ ${ }^{2}$ Núcleo de Informática Aplicada a Educação (NIED) - Universidade Estadual de \\ Campinas (UNICAMP) \\ Campinas - SP - Brasil \\ andre.constantinodifsp.edu.br, \{farantes, ffreire\} @unicamp.br, \\ daniel.realuno.ifsp.edu.br
}

\begin{abstract}
The number of users who benefit from m-Learning is increasing mainly due to the popularization of mobile devices like smartphones and tablets. The interaction on these devices, performed by touch, pen or gestures, it is different from the interaction that occurs on desktop computers. Due to the diversity of computing devices, and considering the plurality of student devices, it is also necessary that educational tools be multi-device. In this article, we present the EdiMM tool, a multi-semiotic Web text editor with multimodal interaction and for multi-device, supporting individual and collaborative work.
\end{abstract}

Resumo. O número de usuários que se beneficiam do m-Learning está aumentando, principalmente devido a popularização dos dispositivos móveis como smartphones e tablets. A interação nesses dispositivos, realizada por toque, a caneta ou por gestos, se diferente da interação ocorrida em computadores de mesa. Devido a diversidade de dispositivos computacionais, e considerando a pluralidade de dispositivos dos alunos, é necessário também que ferramentas educacionais sejam multidispositivos. Apresentamos nesse artigo a ferramenta EdiMM, um editor de texto multissemiótico para a Web com interação multimodal e para multidispositivos, apoiando o trabalho individual e colaborativo.

\section{Introdução}

Com a popularização dos dispositivos móveis, equipamentos dotados de autonomia e leveza, o que possibilita que sejam facilmente transportados, e das redes sem fio, começam a surgir o uso desses dispositivos com propósitos educacionais. Sung et al. (2005) definem m-Learning (do inglês mobile learning, que significa aprendizagem móvel) como qualquer tipo de educação que acontece quando o aprendiz não tem uma localização geográfica fixa ou pré-determinada; ou aquela que acontece quando o aprendiz aproveita as oportunidades de aprendizado oferecidas pelas tecnologias 
VIII Congresso Brasileiro de Informática na Educação (CBIE 2019)

Anais dos Workshops do VIII Congresso Brasileiro de Informática na Educação (WCBIE 2019)

móveis. Os dispositivos móveis permitem ao aluno estudar em qualquer lugar (anywhere) e a qualquer hora (anytime).

O EdiMM é uma aplicação multidispositivo de código livre para apoiar a elaboração de textos multissemióticos com interação multimodal, ou seja, explora as diferentes modalidades de interação. Uma das premissas de desenvolvimento do EdiMM é possibilitar que o seu uso seja realizado por um amplo conjunto de dispositivos computacionais, principalmente os acessíveis aos alunos. $\mathrm{O}$ objetivo deste artigo é apresentar o EdiMM, enfocando suas três características: multissemiótico, multimodal e multidispositivo. $\mathrm{Na}$ Seção 2 apresentamos as definições de multissemiótico e multimodal. Na Seção 3 apresentamos o EdiMM. Na Seção 4, encerramos o artigo com considerações finais e trabalhos futuros.

\section{Fundamentação Teórica}

Segundo Arantes et al. (2017), os textos que agregam texto escrito e imagens, ainda hoje fartamente utilizados em jornais, revistas, propagandas são exemplos de textos multissemióticos mais comuns, e também são os exemplos mais antigos. Citando Dionísio (2006), os autores descrevem que a interpretação e produção de textos multissemióticos requerem, além de domínio da escrita tradicional, outras habilidades ou multiletramentos, necessitando aprender a atribuir sentido articulando diferentes tipos de informação: texto verbal, vídeo, arquivos de som, imagem etc.

Citando Braga e Ricarte (2005), Arantes et al. afirmam que o texto multissemiótico afeta não só as práticas sociais que envolvem a comunicação, mas também promove mudanças na própria linguagem. Os autores postulam que "de forma análoga a que aconteceu no passado remoto, quando o homem inventou diferentes tipos de escrita até chegarmos ao alfabeto tal como o conhecemos, é possível que no futuro a elaboração de textos multissemióticos seja uma atividade corriqueira, isto é, possível de ser feita tal como hoje escrevemos à mão ou digitamos em nosso laptop" e afirmam que mais lemos textos multissemióticos do que, efetivamente, produzimos textos desse tipo.

Modalidade é o termo empregado para definir o modo como uma entrada do usuário e uma saída do sistema são expressas. O modo de comunicação se refere ao canal de comunicação usado por duas entidades diferentes que interagem [Alty e McCartney 1991]. Nigay e Coutaz (1995) definem modalidade como um método de interação que um agente pode usar para alcançar uma meta. Segundo os autores uma modalidade pode ser especificada em termos gerais como "usando fala" ou em termos mais específicos como "usando microfone". Bernsen (2008) afirma que duas modalidades não são equivalentes, pois as modalidades diferem uma da outra em relação aos pontos fortes e fracos de expressividade e também em sua relação aos sistemas perceptual, cognitivo e emocional do ser humano.

\section{O EdiMM}

O EdiMM [Freire et al. 2015] [Arantes et al. 2017] - Editor de Texto Multissemiótico e Multimodal - é uma ferramenta computacional que visa auxiliar a construir textos multissemióticos com interação multimodal e para multidispositivos. O EdiMM é composto por uma tela (Figura 1) que possibilita ao usuário escolher dentre diversas ferramentas como a inserção de imagens, a escrita manuscrita, a escrita digitada, desenho de figuras geométricas etc. As ferramentas estão dispostas à esquerda da tela, 
VIII Congresso Brasileiro de Informática na Educação (CBIE 2019)

Anais dos Workshops do VIII Congresso Brasileiro de Informática na Educação (WCBIE 2019)

enquanto que os atributos para a ferramenta selecionada são exibidos ao topo, em conjunto com funcionalidade para mover, apagar, salvar, configurar e acionamento de funcionalidades por toque.

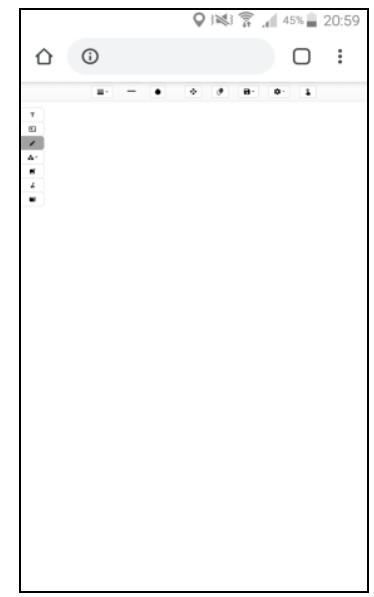

(a)

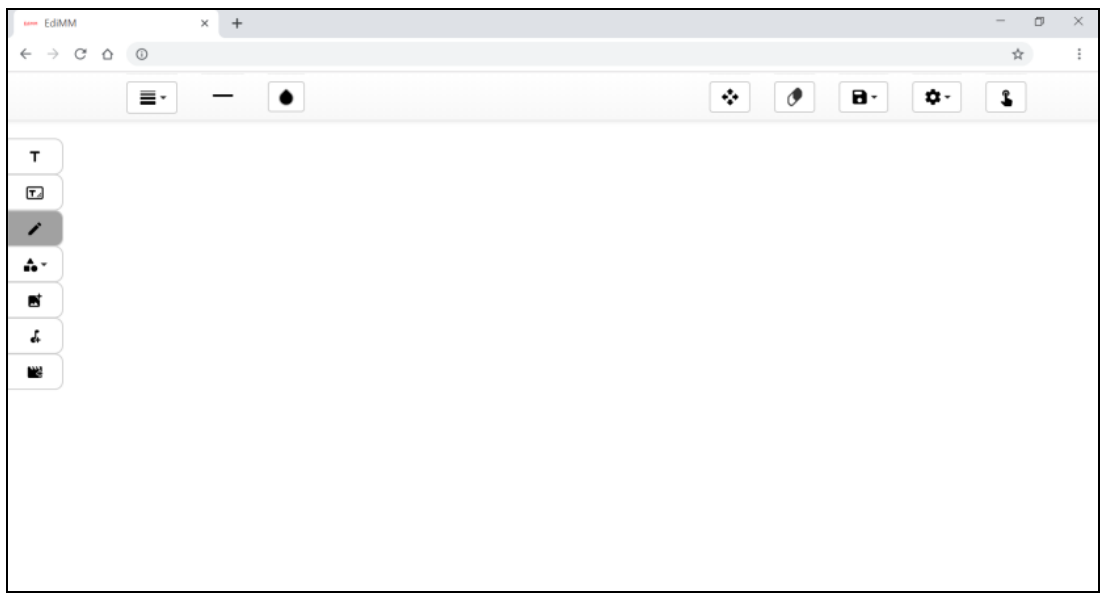

(b)

Figura 1. A interface de usuário do EdiMM visualizada (a) em dispositivo móvel e (b) em tablet ou computador de mesa.

Dentre as funcionalidades do EdiMM, destacamos:

Inserção de imagens, sons e vídeos: caso o usuário esteja usando um computador convencional, este poderá selecionar uma imagem, som ou um vídeo na unidade de armazenamento. Caso esteja usando um dispositivo móvel, poderá escolher em selecionar uma imagem, som ou vídeo da unidade de armazenamento ou tirar uma foto ou vídeo com a câmera do celular ou gravar um som com o microfone do dispositivo;

Interação por toque: caso o usuário esteja usando um dispositivo com tela sensível ao toque, o usuário pode acionar esta funcionalidade para utilizar seus dedos para acionar as ferramentas e alterar o documento com o dedo. Necessário acionar para modificar o comportamento do navegador na interação por toque. Pretende-se, no futuro, automatizar o reconhecimento do dispositivo do usuário de modo que o acionamento de interação por toque seja automático caso o usuário utilize um dispositivo com tela sensível ao toque;

Interação por caneta: caso o usuário esteja utilizando um dispositivo com tela sensível à caneta, este poderá utilizar a caneta para acionar as ferramentas e alterar o documento.

Decidiu-se que o EdiMM seria uma ferramenta Web e a adoção das tecnologias HTML, JavaScript e CSS para a implementação da interface de usuário (componente cliente da arquitetura adotada) e a linguagem PHP para o código do servidor. Ressaltase que a adoção dessas tecnologias é importante para a ferramenta ser multidispositivo, evitando que seja necessário desenvolver uma versão para cada dispositivo ou plataforma e, também, que a ferramenta possa ser integrada a Ambientes Virtuais de Aprendizagem. Em relação a multidispositivos, optou-se por aplicar técnicas de Design Responsivo [Marcotte 2011] de modo que o editor possa ser utilizado em diferentes dispositivos, como computadores desktop, laptops e dispositivos móveis como tablets e celulares. 
VIII Congresso Brasileiro de Informática na Educação (CBIE 2019)

Anais dos Workshops do VIII Congresso Brasileiro de Informática na Educação (WCBIE 2019)

Além de testes realizados em computadores de mesa, laptops, smartphones e tablets, o EdiMM também é funcional em lousas digitais. A lousa digital, equipamento computacional sensível à caneta para uso pedagógico com capacidade de aproveitar várias mídias é um auxílio digital para os professores ao tornar as aulas mais interativas o que proporciona uma aula com apresentação que possibilita utilizar fontes trazendo imagens, vídeos e figuras para enfatizar o assunto tratado e a preparação de todo conteúdo didático.

Após um processo de identificação e correção de problemas de usabilidade utilizando o EdiMM em uma lousa digital, um estudo de caso foi executado para investigar o uso do EdiMM em sala de aula em uma aula expositiva na qual o professor explica um determinado conceito. O laboratório que ocorreu o estudo de caso é equipado com 20 computadores de mesa para os alunos, mesa para o professor e um quadro branco de vidro temperado fixado na parede, no qual a lousa digital projetava enquanto que um outro projetor projetava em uma tela de projeção (Figura 2). O código fonte em linguagem $\mathrm{C}$ referente ao conteúdo foi projetado na tela de projeção, enquanto que a lousa digital foi utilizada para explicação do estado da memória durante execução do código. Somente o professor interagiu com o EdiMM. Analisando a Figura 2, percebe-se que o usuário (o professor) utilizou basicamente a ferramenta de texto manuscrito escrevendo com diferentes cores para explicar o funcionamento da estrutura de dados fila em memória sequencial da disciplina de Estrutura de Dados. O professor também utilizou a borracha e a ferramenta de movimentação de objetos.

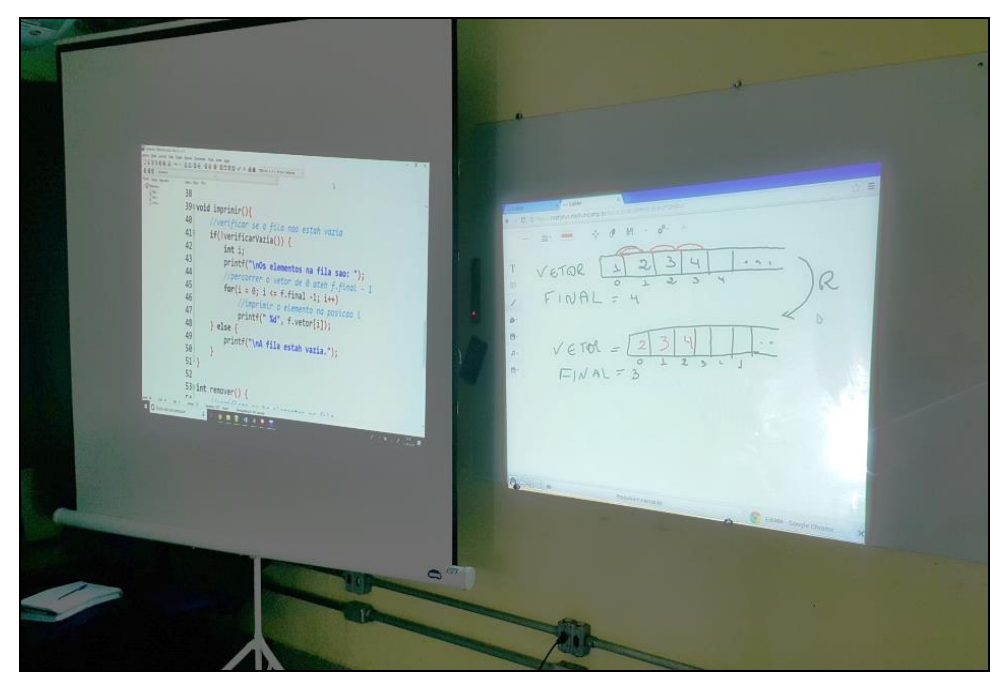

(a)

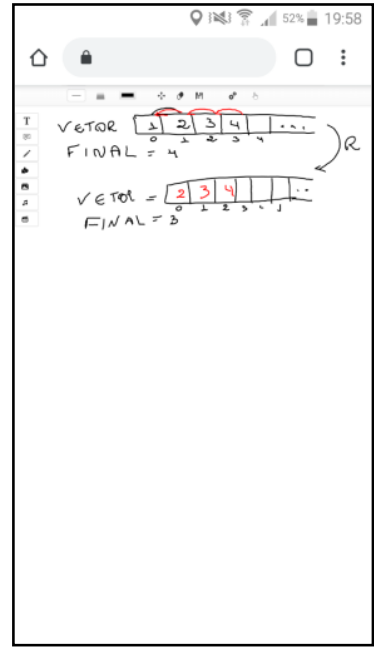

(b)

Figura 2. Utilização do EdiMM em um estudo de caso (a) projeção com a lousa digital e (b) visualização em um dispositivo móvel.

Para possibilitar que diversos usuários, em dispositivos diferentes, possam manipular o mesmo espaço digital de produção, desta forma apoiando o trabalho colaborativo, foi adicionado ao código a comunicação via WebSockets. Um usuário, ao acessar a ferramenta, é adicionado a sessão daquele documento e, quando ele ou outro usuário alteram o documento, os dados são enviados ao servidor que fica responsável por encaminhar os dados a todos os usuários naquela sessão. 
VIII Congresso Brasileiro de Informática na Educação (CBIE 2019)

Anais dos Workshops do VIII Congresso Brasileiro de Informática na Educação (WCBIE 2019)

\section{Considerações Finais}

Com a popularização dos dispositivos móveis, começam a surgir o uso desses dispositivos com propósitos educacionais. Pensando na tecnologia dos computadores convencionais e na dos dispositivos móveis, acreditamos em uma complementação de uso dessas tecnologias. Dependendo da tarefa a ser realizada, utilizar um determinado hardware será mais rápido, eficiente e/ou oferecerá uma experiência mais gratificante aos usuários. É com esse pensamento que desenvolvemos o EdiMM (Editor de Texto Multissemiótico e Multimodal), no qual as diversas tecnologias (e as diferentes modalidades que essas tecnologias dão suporte) são utilizadas em prol das experiências dos usuários e do planejamento e realização das atividades de ensino e aprendizado. Planejamos para a próxima versão da ferramenta o suporte a interação por voz e por gestos, modalidades suportadas pelos periféricos disponíveis nos dispositivos móveis.

\section{Agradecimentos}

Agradecemos ao CNPq e ao IFSP pelas bolsas de iniciação tecnológica, modalidade PIBITI, cujo projetos visaram o desenvolvimento da ferramenta.

\section{Referências}

Alty, J. and McCartney, C. (1991). Design of a Multi-Media Presentation System For A Process Control Environment, In Eurographics Multimedia Workshop: Session 8: Systems, Stockholm, Suécia.

Arantes, F. L., Freire, F. M. P., Breuer, J., Silva, A. C. da, Oliveira, R. C. A., e Vascon, L. E1. L. (2017). "Towards a Multisemiotic and Multimodal Editor", Journal of Computer Science and Technology, v. 17, p. 100-109.

Bernsen, N. O. (2008). "Multimodality Theory", Multimodal User Interfaces: From signal to interaction, D. Tzovaras, Berlim, Alemanha, Springer-Verlag Berlin Heidelberg, p. 5-28.

Braga, D. B. e Ricarte, I. (2005). Letramento e Tecnologia. Cefiel \& MEC, Secretaria de Ensino Fundamental.

Dionisio, A. P. (2006). "Gêneros multimodais e multiletramentos", Gêneros textuais, reflexões e ensino, A. M. Karwoski, B. Gaydeczka, e K. S. Brito, Rio de Janeiro, Lucerna, p. 131-143.

Freire, F. M., Arantes, F. L., Silva, A. C. da, e Vascon, L. E. L. (2015). Estudo de viabilidade de um Editor Multimodal: o que pensam os alunos?, In Anais do XX Congreso Internacional de Informática Educativa, Santiago, Universidad de Chile, Facultad de Ciencias Físicas y Matemáticas, v. 11, p. 109-119.

Marcotte, E. (2011). Responsive Web Design, A Book Apart.

Nigay, L., e Coutaz, J. (1995). A Generic Platform for Addressing the Multimodal Challenge, In Proceedings of $13^{\text {th }}$ SIGCHI Conference on Human Factors in Computing Systems, Colorado, EUA, p. 98-105.

Sung, M., Gips, J., Eagle, N., Madan, A., Caneel, R., Devaul, R., Bonsen, J., e Pentland, A. (2005). Mobile-IT Education (MIT. EDU): m-learning applications for classroom settings. In Journal of Computer Assisted Learning, v. 21, p. 229-237. 